\title{
COVID-19 pandemic: personal view to a new model of pediatric practice
}

\author{
Zakaria Barsoum $^{1}$
}

Received: 12 May 2020 / Accepted: 25 May 2020 / Published online: 10 June 2020

(c) Children's Hospital, Zhejiang University School of Medicine 2020

The coronavirus pandemic is an unprecedented challenge to health care professionals. Central in its management remain social distancing, personal hygiene and wearing proper personal protective equipments. Distant medical practising is alien to clinicians. Virtual consultations deploying recent technology have now replaced the normal practice of routine clinics.

Virtual consultations minimise the risk of COVID-19 transmission, promote public protection and reduce the backlog of waiting lists during this time of testing.

Many clinicians may feel uncomfortable with this new model of practice where the gold standard rule in medical practice is direct contact with patients. Face to face consultations help build up rapport between doctors and patients. Face to face clinical practice enables clinicians to better identify clinical problems and provide meticulous clinical assessment. Uplifting patient safety and sound clinical judgement are a top priority for physicians.

Virtual clinics are useful tools at the time of COVID-19 pandemic when health care demands are pressing. Clinicians are at the front door in this battle and subsequently are at increased risks of contracting disease and transmitting it to their close contacts and loved ones, placing physicians under immense emotional pressures.

Children with allergy such as food allergy are a special cohort that may benefit well from virtual consultations. The preponderance of them are well and parents can provide a detailed allergy focused clinical history guided by clinicians during virtual consultations. The quality of video recording can provide clues about various allergic skin manifestations. Obtaining allergy focused clinical history is a quality statement [1].

In our unit, paediatric allergy team had forethought to launch virtual clinics before recent recommendations from

Zakaria Barsoum

doctorzakaria@yahoo.com

1 South West Acute Hospital-Paediatric Department, Northern Ireland, 124 Irvinestown Rd, Enniskillen BT 74 6DN, UK
The British Society for Allergy and Clinical Immunology (BSACI) justified this model [2]. Service started on March 23, 2020, eight clinics were run and 50 patients reviewed. No adverse events were reported and patient perception of the new service was encouraging.

Adjustments were made related to specific allergy investigations such as skin prick tests, congruent with BSACI recommendations, those tests can be deferred during COVID19 pandemic [3].

In brief, virtual clinics are useful at times of pandemics although they lack direct clinical relationship with patients. Virtual consultations deploy recent technology in medicine and are recommended by professional bodies. Although clinical confidence and appropriateness of use may vary in various aspects of clinical care, our experience in paediatric allergy is satisfactory.

Author contributions $\mathrm{BZ}$ wrote, revised and approved the final version of the manuscript.

Funding None.

\section{Compliance with ethical standards}

Ethical approval Not needed.

Conflict of interest Nothing to declare.

\section{References}

1. The National Institute for Health and Care Excellence (NICE). NICE report. March 2016. https://www.nice.org.uk. Accessed 24 Mar 2016.

2. BSACI report. https://bsaci.org. Accessed 24 Mar 2020.

3. Shaker MS, Oppenheimer J, Grayson M, Stukus D, Hartog N, Hsieh EWY, et al. COVID-19: pandemic contingency planning for the allergy and immunology clinic. J Allergy Clin Immunol Pract. 2020;8:1477-88.e5.

Publisher's Note Springer Nature remains neutral with regard to jurisdictional claims in published maps and institutional affiliations. 